\title{
Therapeutic Effects of Add-On Tenapanor for Hemodialysis Patients with Refractory Hyperphosphatemia
}

\author{
Takashi Shigematsu $^{\mathrm{a}}$ Yotaro Une $^{\mathrm{b}}$ Kazuaki lkejiri $^{\mathrm{b}}$ Hironori Kanda $^{\mathrm{b}}$ \\ Masafumi Fukagawa ${ }^{c}$ Tadao Akizawa $^{d}$

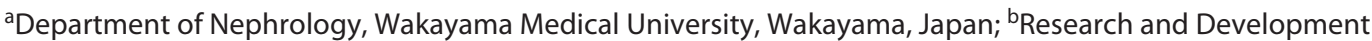 \\ Division, Kyowa Kirin Co., Ltd., Tokyo, Japan; 'Division of Nephrology, Department of Internal Medicine,

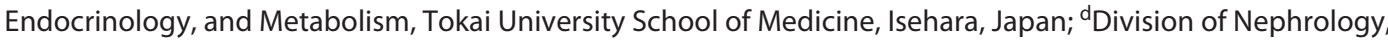 \\ Department of Medicine, Showa University School of Medicine, Tokyo, Japan
}

\section{Keywords}

Tenapanor $\cdot \mathrm{Na}+/ \mathrm{H}+$ antiporter $3 \cdot$ Serum phosphorus level $\cdot$ Hyperphosphatemia $\cdot$ Hemodialysis

\begin{abstract}
Introduction: Phosphate binders are used to treat hyperphosphatemia. Some patients have inappropriately controlled serum phosphorus levels, which may occur for many reasons, including a high pill burden and adverse events (AEs). Tenapanor selectively inhibits the passive paracellular transfer of phosphate in the gastrointestinal tract, thereby reducing serum phosphorus levels. This novel mechanism of action may contribute to improved phosphate management. The efficacy and safety of tenapanor have not been evaluated in Japanese patients with high serum phosphorus levels despite treatment with phosphate binders. This study aimed to assess the efficacy and safety of add-on tenapanor therapy for reducing serum phosphorus levels in this population. Methods: This multicenter, double-blind, randomized, placebo-controlled trial enrolled patients with refractory hyperphosphatemia undergoing hemodialysis. Patients were randomly assigned in a 1:1 ratio to receive tenapanor or placebo as an add-on to their phosphate binder regimen
\end{abstract}

karger@karger.com www.karger.com/ajn

Karger!
C 2021 The Author(s)

Published by S. Karger AG, Basel

This is an Open Access article licensed under the Creative Commons Attribution-NonCommercial-4.0 International License (CC BY-NC) (http://www.karger.com/Services/OpenAccessLicense), applicable to the online version of the article only. Usage and distribution for commercial purposes requires written permission. for 6 weeks. Change in serum phosphorus levels at week 6 (day 43) compared with the baseline value (day 1 , week 0 ) (primary endpoint), achievement of target serum phosphorus levels (serum phosphorus level $\leq 6.0$ or $\leq 5.5 \mathrm{mg} / \mathrm{dL}$ ), and safety, based on all AEs and drug-related AEs, were among the outcomes evaluated. Results: In total, 24 patients were randomly assigned to the placebo group and 23 to the tenapanor group. The mean serum phosphorus level decreased from $7.01 \mathrm{mg} / \mathrm{dL}$ on day 1 to $6.69 \mathrm{mg} / \mathrm{dL}$ on day 43 in the placebo group and from $6.77 \mathrm{mg} / \mathrm{dL}$ on day 1 to $4.67 \mathrm{mg} / \mathrm{dL}$ on day 43 in the tenapanor group. In the placebo and tenapanor groups (modified intent-to-treat population), the mean (standard deviation) change in the serum phosphorus level at day 43 (last observation carried forward [LOCF]) was $0.08(1.52) \mathrm{mg} / \mathrm{dL}$ and $-1.99(1.24) \mathrm{mg} / \mathrm{dL}$, respectively, with a between-group difference of -2.07 ( $95 \%$ confidence interval: $-2.89,-1.26 ; p<0.001$ ). The target achievement rate (serum phosphorus level $\leq 6.0 \mathrm{mg} / \mathrm{dL}$ at week 6 [LOCF]) was 37.5 and $87.0 \%$ in the placebo and tenapanor groups, respectively. Diarrhea was the most common drug-related $A E$, and it occurred in 8.3 and $65.2 \%$ of patients in the placebo and tenapanor groups, respectively. No specific AEs were observed with add-on tenapanor or with phosphate binders. Discussion/Conclusion: Therapy with existing phosphate binders 
and add-on tenapanor resulted in a significant decrease in serum phosphorus level compared with the placebo group in patients with refractory hyperphosphatemia despite treatment with phosphate binders. No new safety signals were raised, and add-on tenapanor was generally well tolerated.

(c) 2021 The Author(s)

Published by S. Karger AG, Basel

\section{Introduction}

The global burden of CKD is considerable, with a global prevalence of $9.1 \%$. In 2017, an estimated 2.6 million individuals died worldwide from CKD and its effects on cardiovascular disease [1]. With disease progression, CKD eventually results in several metabolic complications, including hyperphosphatemia [2], and the importance of maintaining phosphorus homeostasis to reduce the risk of cardiovascular events and mortality in patients with CKD is well recognized [3-5]. However, dietary restrictions and adequately prescribed hemodialysis are generally insufficient to maintain phosphate homeostasis, and most patients require management with phosphate binders [6].

The Japanese Society for Dialysis Therapy (JSDT) guideline [7] recommends the individualized selection of phosphate binders, either as monotherapy or in combination for the management of hyperphosphatemia in CKD patients. Thus, the serum phosphorus level is controlled within the target range $(3.5-6.0 \mathrm{mg} / \mathrm{dL})$ recommended in the guideline [7] in approximately $70-80 \%$ of dialysis patients. According to the JSDT guideline, the control achievement rate has remained unchanged in recent years, with approximately $20-30 \%$ of patients exceeding the upper limit of the target range, indicating inadequate phosphorus control among these patients.

Although the efficacy of phosphate binders has been confirmed, some patients are likely not receiving proper treatment because they experience issues, such as adverse events (AEs) and high pill burden [6], which may hinder phosphate binder efficacy and affect adherence to treatment [8]. As such, those patients' serum phosphorus levels exceed the upper limit of the target range. Further, monotherapy and combination therapy with phosphate binders, which have similar mechanisms, may have some limitations. Therefore, a drug that acts via a novel mechanism of action for managing hyperphosphatemia is needed.

Tenapanor is a novel selective $\mathrm{Na}+\mathrm{H}+$ antiporter 3 (NHE3) inhibitor formulated as a small (ranging from 5 to $12 \mathrm{~mm}$ ) and round tablet. On the luminal side of intestinal epithelial cells, it inhibits the sodium-hydrogen exchange, causing the accumulation of intracellular protons and lowering the $\mathrm{pH}$ in epithelial cells. This decline in intracellular $\mathrm{pH}$ inhibits the passive paracellular diffusion of phosphorus and reduces the serum phosphorus level. The accumulation of sodium ions in the intestinal tract increases the amount of water in the intestinal tract, resulting in loose stools [9]. A phase I study in healthy Japanese adults found that tenapanor increased fecal excretion of sodium and phosphorus and decreased their urinary excretion [10]. In a phase III study of tenapanor in hemodialysis patients with hyperphosphatemia, tenapanor (3- and 10-mg fixed doses and 30-mg down-titration dose) significantly reduced serum phosphorus levels, compared with placebo. Diarrhea occurred in 34 of 71 patients (47.9\%) in the $30-\mathrm{mg}$ tenapanor group [11]. The AMPLIFY study confirmed the efficacy and safety of tenapanor in combination with phosphate binders as a dual-mechanism approach for hyperphosphatemia therapy in patients with CKD on dialysis [12].

The efficacy of tenapanor has not been evaluated in Japanese hemodialysis patients with inadequate phosphorus management. The present study aimed to assess the efficacy of tenapanor for reducing serum phosphorus levels and its safety during add-on treatment with conventional phosphate binders for Japanese hemodialysis patients with refractory hyperphosphatemia compared with placebo. We assessed changes in serum phosphorus levels from baseline and the achievement rate of serum phosphorus levels below $6.0 \mathrm{mg} / \mathrm{dL}$ with conventional phosphate binders and add-on tenapanor.

\section{Materials and Methods}

\section{Study Design, Randomization, and Intervention}

This was a multicenter, double-blind, randomized, placebocontrolled trial conducted at 9 institutions in Japan between April 2019 and December 2019. The study had 3 periods: a screening period, an observation period of 2-3 weeks (including enrollment and randomization), and an evaluation (administration) period of 6 weeks (Fig. 1a). Subjects were randomly assigned to either the tenapanor or placebo group in a 1:1 ratio, using a dynamic allocation method. The allocation factors were serum phosphorus level (6.1-8.0 $\mathrm{mg} / \mathrm{dL}$ and $8.1-9.9 \mathrm{mg} / \mathrm{dL}$ ) at the time of enrollment and study site. The random allocation sequence was generated by Bellsystem 24, Inc. (Tokyo, Japan). The principal investigators at each site enrolled subjects using an Interactive Web Response System (IWRS). Because the study was double-blind, the sponsor, health care provider (including the principal investigators and clinical research coordinator), and subjects were blinded to treatment and the drug numbers issued by the IWRS. 


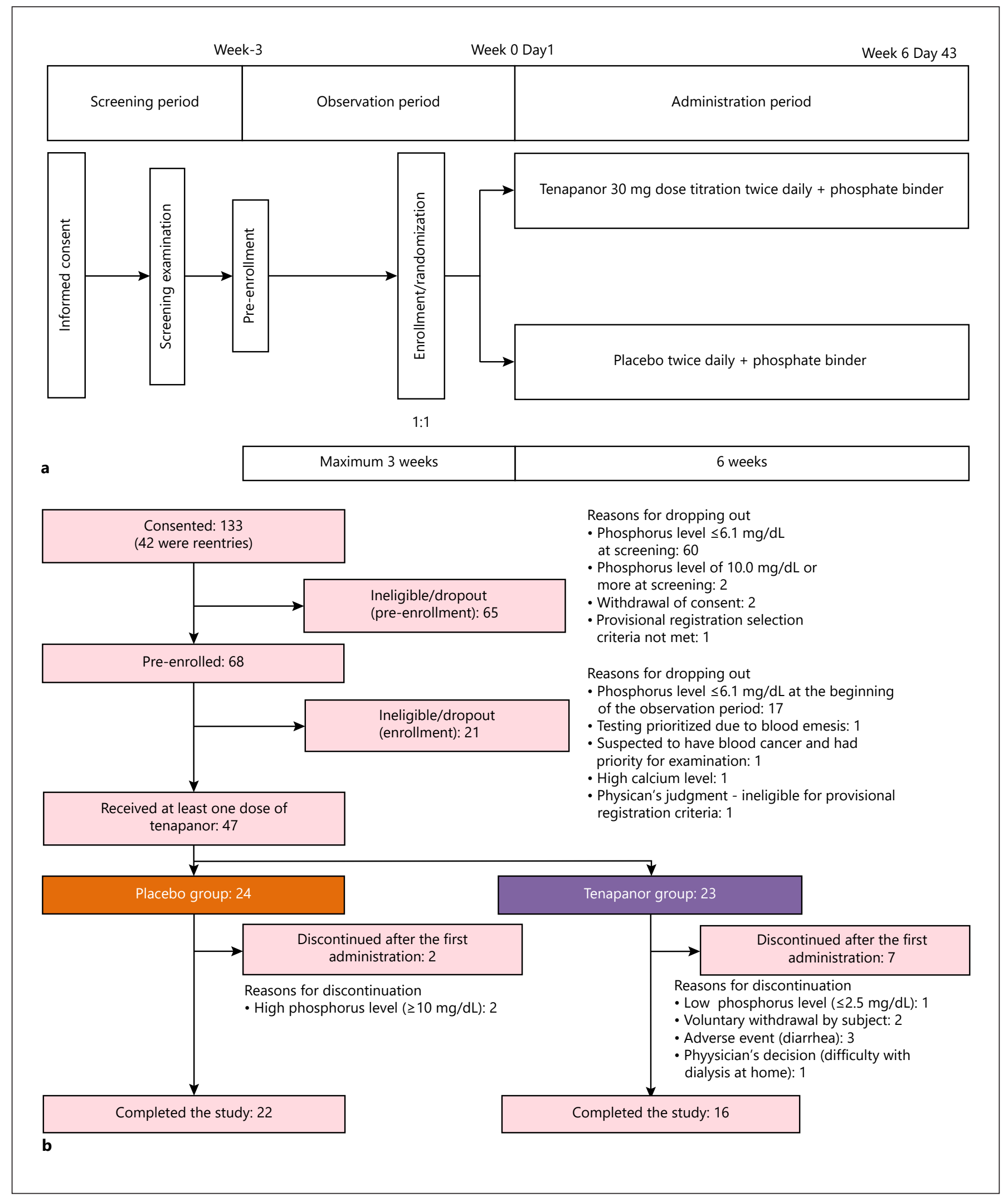

Fig. 1. a Study design. b Patient disposition. 
Placebo or tenapanor was administered for 6 weeks. In principle, tenapanor was taken orally twice daily (BID), immediately before meals (breakfast and dinner). Treatment, at a dosage of $30 \mathrm{mg}$ BID, began shortly before the meal, following the end of dialysis after the longest dialysis interval. All subjects were monitored weekly for gastrointestinal symptoms to determine the need for dose adjustments. The dose was adjusted immediately before the meal after completion of dialysis, after the maximum dialysis interval, if the gastrointestinal symptoms were considered to have been caused by the investigational products and based on the physician's judgment. Dose reductions were made 1 step at a time from $30 \mathrm{mg}$ BID, $20 \mathrm{mg}$ BID, and $10 \mathrm{mg}$ BID to $5 \mathrm{mg} \mathrm{BID}$, up to a maximum of 3 times. If further dose reductions were deemed necessary, the study treatment was discontinued. Restricted concomitant medications, namely, phosphate binders, and therapies were administered continuously during the study at the preexisting dose before the start of the study.

Prohibited concomitant treatments and therapies were drugs for hyperphosphatemia treatment (from the beginning of the observation period until week 6), other than the phosphate binders in the preexisting regimen, and peritoneal dialysis (prohibited from 13 weeks before screening until week 6). If the patient was receiving vitamin $\mathrm{D}$ replacement therapy or calcimimetics, the dosage and administration were maintained constant from 2 weeks before the screening period to enrollment. In principle, the dosage and administration remained unchanged. However, if AEs occurred for which a causal relationship with the phosphate binder could not be ruled out, the dose could be reduced or discontinued. If the AE was resolved, improved, or trended to resolution by reduction or discontinuation of the dose, the dose could be restored to the dose at the beginning of treatment. Hemodialysis was allowed, but no changes in dialysis conditions were permitted, other than dry weight, from 2 weeks before the screening period until the final evaluation at week 6. Dietary phosphorus restrictions were allowed, but no changes in the diet were permitted until the final evaluation at week 6 .

In general, data were collected in electronic case report forms, but data on medication adherence, date and time of defecation, and Bristol Stool Form Scale (BSFS) score were collected in patient diaries. The BSFS is a diagnostic tool designed to classify human feces by its form into 7 categories. Types 1 and 2 indicate constipation, with 3 and 4 being the ideal stool forms, which can be easily defecated as it does not contain excess liquid. Type 5 indicates a lack of dietary fiber, and types 6 and 7 indicate diarrhea. General biochemical tests, serum phosphorus level, serum calcium level, and calcium-phosphorus product ( $\mathrm{Ca} \times \mathrm{P}$ product) were measured weekly. Intact parathyroid hormone (iPTH) was measured every 2 weeks. Intact fibroblast growth factor 23 (iFGF23) was evaluated at the screening examination and weeks 0 and 6 . The investigators (and their institutions), patients, and sponsor were blinded to the results of $\mathrm{P}, \mathrm{iPTH}$, and FGF23.

The ethical review boards of the participating sites approved the protocol and related documents. The study adhered to Good Clinical Practice guidelines and the Declaration of Helsinki, as well as local laws and regulations. All patients provided informed consent before the conduct of any study procedures. The study was registered in Clinicaltrials.gov under the identifier NCT03864445.

\section{Patients}

The main eligibility criteria were as follows: male or female patients aged $\geq 20-<80$ years at the time of informed consent with stable CKD and who have undergone hemodialysis 3 times per week for at least 12 weeks until the screening period; with constant dialysis conditions, other than dry weight, for 2 weeks before the screening period and 3 weeks of the observation period after the provisional enrollment; taking phosphate binders 3 times daily, with a constant dosage and administration from 2 weeks before the screening period until enrollment; with serum phosphorus levels of $\geq 6.1$ and $<10.0 \mathrm{mg} / \mathrm{dL}$ at the time of the screening period and after 1 or 2 weeks of observation; and $\geq 1.2 \mathrm{kt} / \mathrm{V}$ urea in the most recent routine medical examination prior to the screening period. The main exclusion criteria were as follows: iPTH $>600 \mathrm{pg} / \mathrm{mL}$; history of inflammatory bowel disease or diarrhea-predominant irritable bowel syndrome; and presence of diarrhea or loose stools, defined as BSFS $\geq 6$ and number of defecations $\geq 3$ per day for 2 or more days, within 1 week before enrollment.

\section{Study Endpoints}

The primary endpoint was change in serum phosphorus levels at 6 weeks (day 43) after treatment initiation compared with the baseline value (day 1 , week 0 ). The secondary endpoints were changes in the serum phosphorus level at each time point after the administration of tenapanor compared with the baseline value, attainment of target serum phosphorus levels (serum phosphorus level $\leq 6.0$ [7] or $\leq 5.5 \mathrm{mg} / \mathrm{dL}$ ), and changes in the $\mathrm{Ca} \times \mathrm{P}$ product and serum corrected $\mathrm{Ca}$ levels from baseline to each time point. We examined serum phosphorus level $\leq 5.5 \mathrm{mg} / \mathrm{dL}$ to allow for comparisons with overseas data [13]. The exploratory endpoints were changes from baseline in serum levels of iFGF23 and iPTH.

Safety evaluations were based on all recorded AEs and drugrelated AEs that occurred or worsened between the start of treatment and the end of week 6 (or discontinuation) by the treatment group. The frequencies of AEs were tabulated by preferred term and system organ class using MedDRA version 22.1. Changes in the average BSFS score and the average number of defecations per week were assessed. Changes in laboratory test results (biochemistry and hematology), vital signs, and 12-lead electrocardiogram were also evaluated.

\section{Statistical Analysis}

The target sample size was not determined based on statistical evidence. However, a sample size of 20 patients per group was estimated, assuming that the mean changes in serum phosphorus levels from baseline values at week 6 were $-0.5 \mathrm{mg} / \mathrm{dL}$ and -1.5 $\mathrm{mg} / \mathrm{dL}$, in the placebo and tenapanor groups, respectively, with a standard deviation (SD) of $2.0 \mathrm{mg} / \mathrm{dL}$ in both groups.

The modified intent-to-treat (mITT) population included all randomized patients, except those who did not receive the study drug and those lacking serum phosphorus measures from the start of the study. The safety analysis set included all subjects eligible for enrollment, except patients who had never received the study drug.

Categorical data were summarized in frequencies and percentages, and continuous data were summarized as the number of subjects, mean and SD, and minimum, median, and maximum. For the analysis of the primary endpoint, the $t$ test was used for comparison between placebo and tenapanor groups. If a serum phosphorus level at week 6 was missing, the serum phosphorus level at the time of final measurement was used for analysis according to the last observation carried forward (LOCF) method. A 2-sided $p$ value of 0.05 was used as the significance level. A sensitivity analysis was performed using the mixed-effects model for repeated mea- 
Table 1. Background characteristics of patients in the placebo and tenapanor groups

\begin{tabular}{|c|c|c|}
\hline Parameter & $\begin{array}{l}\text { Placebo } \\
(N=24)\end{array}$ & $\begin{array}{l}\text { Tenapanor } 30 \mathrm{mg} \text { dose } \\
\text { titration }(N=23)\end{array}$ \\
\hline \multicolumn{3}{|l|}{ Sex } \\
\hline Female & $9(37.5)$ & $4(17.4)$ \\
\hline Male & $15(62.5)$ & $19(82.6)$ \\
\hline Age (mean $[S D]$ ), years & $59.4(9.2)$ & $62.1(8.5)$ \\
\hline Height, $\mathrm{cm}$ & $164.09(8.38)$ & $165.81(8.52)$ \\
\hline Weight on the day before dialysis (mean [SD]), $\mathrm{kg}$ & $68.27(16.97)$ & $65.27(9.25)$ \\
\hline Weight on the day after dialysis (mean $[\mathrm{SD}]$ ), $\mathrm{kg}$ & $64.91(16.50)$ & $62.35(8.97)$ \\
\hline $\mathrm{BMI}($ mean $[\mathrm{SD}]), \mathrm{kg} / \mathrm{m}^{2}$ & $25.14(4.79)$ & $23.71(2.75)$ \\
\hline \multicolumn{3}{|l|}{ Primary disease } \\
\hline Diabetic nephropathy & $9(37.5)$ & $10(43.5)$ \\
\hline Chronic glomerulonephritis & $9(37.5)$ & $10(43.5)$ \\
\hline Nephrosclerosis & $1(4.2)$ & 0 \\
\hline Polycystic kidney & $1(4.2)$ & 0 \\
\hline Chronic pyelonephritis & 0 & 0 \\
\hline Others & $4(16.7)$ & $3(13.0)$ \\
\hline \multicolumn{3}{|l|}{ Phosphate binder at day 1} \\
\hline Calcium carbonate & $16(66.7)$ & $19(82.6)$ \\
\hline Sevelamer hydrochloride & $5(20.8)$ & $4(17.4)$ \\
\hline Lanthanum carbonate & $9(37.5)$ & $5(21.7)$ \\
\hline Bixalomer & $2(8.3)$ & $3(13.0)$ \\
\hline Sucroferric oxyhydroxide & $2(8.3)$ & $3(13.0)$ \\
\hline Ferric citrate hydrate & $8(33.3)$ & $6(26.1)$ \\
\hline \multicolumn{3}{|l|}{ Serum phosphorus level (mean [SD]), mg/dL } \\
\hline At enrollment & $7.38(0.83)$ & $7.18(0.73)$ \\
\hline At baseline & $7.01(1.25)$ & $6.77(0.88)$ \\
\hline \multicolumn{3}{|l|}{ iPTH (mean [SD]), pg/mL } \\
\hline At enrollment & $202.5(161.1)$ & $172.7(116.9)$ \\
\hline At baseline & $174.7(130.4)$ & $168.0(129.9)$ \\
\hline
\end{tabular}

Data in the table are $n(\%)$, unless otherwise indicated. iPTH, intact parathyroid hormone; SD, standard deviation.

sures. In the mixed-effects model for repeated measures, change in serum phosphorus level from baseline was the response variable, the treatment group was the explanatory variable, and the covariates were the evaluation time point, serum phosphorus level on day 1 , and the interaction between the treatment group and the evaluation time point. The statistical software used for the statistical analyses was SAS version 9.4 (SAS Institute Inc., Cary, NC, USA).

\section{Results}

\section{Patient Disposition and Baseline Characteristics}

Forty-seven patients were randomly assigned to the study treatment, 24 to the placebo group and 23 to the tenapanor group, and all patients received at least 1 dose of the study drug. All 47 patients were included in the mITT and safety analysis sets for the primary efficacy and safety assessments. Nine patients discontinued after the first administration of the study drug. In the placebo group, 2 patients discontinued, and both had a phosphorus level $\geq 10 \mathrm{mg} / \mathrm{dL}$. Seven in the tenapanor group discontinued the study. The reasons were AEs ( $n=3$; diarrhea), voluntary withdrawal by the subject $(n=2)$, phosphorus level $\leq 2.5 \mathrm{mg} / \mathrm{dL}(n=1)$, and physician's decision $(n=1)$. Thirty-eight patients completed the study: 22 patients in the placebo group and 16 in the tenapanor group (Fig. 1b).

In both groups, most patients were male (62.5 and $82.6 \%$, respectively) and had a mean age of 59.4 and 62.1 years, respectively. In the placebo and tenapanor groups, the frequency of use of each phosphate binder at baseline in the descending order was as follows: calcium carbonate (66.7 and $82.6 \%$ ), lanthanum carbonate (37.5 and $21.7 \%$ ), ferric citrate hydrate (33.3 and $26.1 \%$ ), sevelamer hydrochloride (20.8 and 17.4\%), and bixalomer and sucroferric acid (both 8.3 and $13.0 \%$ ), respectively. Overall, baseline characteristics in both groups were well balanced (Table 1). 


\section{Study Endpoints}

Primary Endpoint

Mean (SD) serum phosphorus levels by group from day 1 (week 0 ) to the end of study drug administration (day 43, week 6) are shown in Figure 2a. Over time, the mean serum phosphorus level decreased from $7.01 \mathrm{mg} /$ $\mathrm{dL}$ on day 1 to $6.69 \mathrm{mg} / \mathrm{dL}$ on day 43 in the placebo group and from $6.77 \mathrm{mg} / \mathrm{dL}$ on day 1 to $4.67 \mathrm{mg} / \mathrm{dL}$ on day 43 in the tenapanor group.

The mean (SD) change in the serum phosphorus level at day 43 (LOCF) in the placebo and tenapanor groups in the mITT population was $0.08(1.52) \mathrm{mg} / \mathrm{dL}$ and -1.99 (1.24) $\mathrm{mg} / \mathrm{dL}$, respectively, with a between-group difference of -2.07 (95\% confidence interval [CI]: $-2.89,-1.26$; $p<0.001$ ). Roughly, $50 \%$ of subjects in the tenapanor group who completed the study maintained the $30 \mathrm{mg}$ dose.

Mean (SD) changes from day 1 (week 0) to day 43 (week 6) in serum phosphorus levels over time in the mITT population are shown in Figure $2 \mathrm{~b}$. In the placebo group, the mean change from day 1 to day 43 was -0.14 $\mathrm{mg} / \mathrm{dL}$ and that in the tenapanor group was $-1.94 \mathrm{mg} / \mathrm{dL}$.

\section{Secondary Endpoints}

Changes in tenapanor dose and the number of patients who discontinued treatment by week are shown in Figure 2c. Most discontinuations in the tenapanor group occurred at week 0 (mean dose of $30.0 \mathrm{mg}$ ). From week 1 and onward, the number of patients in the tenapanor group, who required reductions of the tenapanor dose, remained constant up to week 5 , at which time the mean dose of tenapanor was $21.6 \mathrm{mg}$.

The target achievement rate of a serum phosphorus level $\leq 6.0 \mathrm{mg} / \mathrm{dL}$ at week 6 (LOCF) was $37.5 \%$ with placebo and $87.0 \%$ with tenapanor and that for a serum phosphorus level $\leq 5.5 \mathrm{mg} / \mathrm{dL}$ at week 6 (LOCF) was $25.0 \%$ with placebo and $73.9 \%$ with tenapanor. The mean (SD) time to achievement of the target serum phosphorus levels $\leq 6.0 \mathrm{mg} / \mathrm{dL}$ and $\leq 5.5 \mathrm{mg} / \mathrm{dL}$ was 20.8 (17.2) and 17.7 (16.8) days with placebo and 4.1 (2.1) and $5.6(4.5)$ days with tenapanor, respectively.

At week 6 (LOCF), the mean changes in the $\mathrm{Ca} \times \mathrm{P}$ product from baseline in the placebo and tenapanor groups were $1.3(12.1) \mathrm{mg} / \mathrm{dL}$ and $-17.5(10.6) \mathrm{mg} / \mathrm{dL}$, respectively. The mean changes in serum corrected $\mathrm{Ca}$ levels from baseline in the placebo and tenapanor groups were $0.15(0.55) \mathrm{mg} / \mathrm{dL}$ and $0.21(0.44) \mathrm{mg} / \mathrm{dL}$, respectively.
Exploratory Analysis

The mean changes and rate of mean change in serum levels of iPTH and iFGF23 in the placebo and tenapanor groups at week 6 are shown in Figure 2d and online suppl. Table 1 (for all online suppl. material, see www.karger. com/doi/10.1159/000516156). At the end of the study, both iPTH and iFGF23 had decreased with tenapanor compared with placebo. No notable changes in sodium, potassium, chloride, magnesium, and bicarbonate were observed (online suppl. Table 1).

\section{Safety}

Overall, in the placebo and tenapanor groups, 9/24 (37.5\%) and 18/23 (78.3\%) patients presented AEs. Only 1 serious AE occurred (4.3\% [1/23]), and this was in the tenapanor group. The event was regarded as a serious and severe $\mathrm{AE}$ and consisted of an ankle fracture (4.3\%), but it was not considered related to the study drug. No deaths occurred during the study. In the placebo and tenapanor groups, there were $8.3 \%(2 / 24)$ and $69.6 \%(16 / 23)$ drugrelated AEs, respectively. Three (13.0\%) patients in the tenapanor group discontinued the study due to drug-related AEs; the AE was diarrhea in all 3 cases (Table 2).

Drug-related AEs in the tenapanor group were all gastrointestinal disorders (16/23 [69.6\%]), specifically diarrhea (15/23 [65.2\%) and nausea, vomiting, and soft feces (each, 1/23 [4.3\%]). Drug-related AEs in the placebo group were 2 cases of diarrhea.

The changes in the average BSFS score and the average number of defecations per week are shown in Figure 2e and $\mathrm{f}$. The increase in BSFS score (Fig. 2e) and the number of defecations (Fig. 2f) was more marked in the tenapanor group at week 1 , which then tapered off to nearly baseline values throughout the study up to week 6 . At week 6 , the mean change in BSFS score was $-0.14(0.75)$ in the placebo group and 1.17 (1.26) in the tenapanor group. The mean (SD) number of weekly defecations was 7.4 (3.3) and 9.1 (5.8) defecations at baseline, 8.7 (5.4) and 12.8 (9.6) defecations at week 1 , and 8.5 (5.0) and 9.6 (4.7) defecations at week 6 in the placebo and tenapanor groups, respectively. There were no significant changes in laboratory test results (biochemistry and hematology), vital signs, or electrocardiography parameters.

\section{Discussion}

In this phase II trial, patients with refractory hyperphosphatemia despite receiving phosphate binders showed a significant reduction in serum phosphorus 

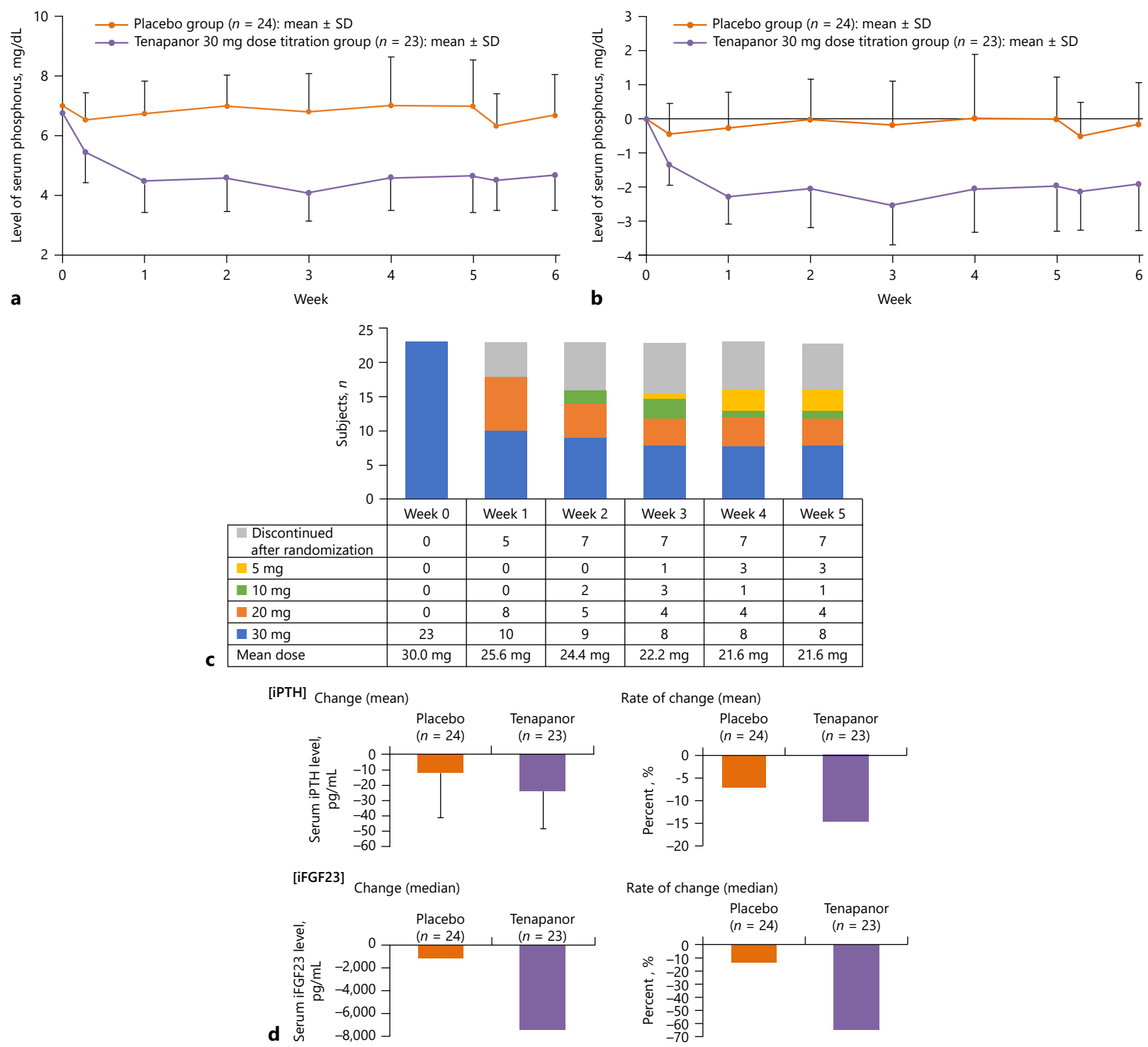

Rate of change (median)
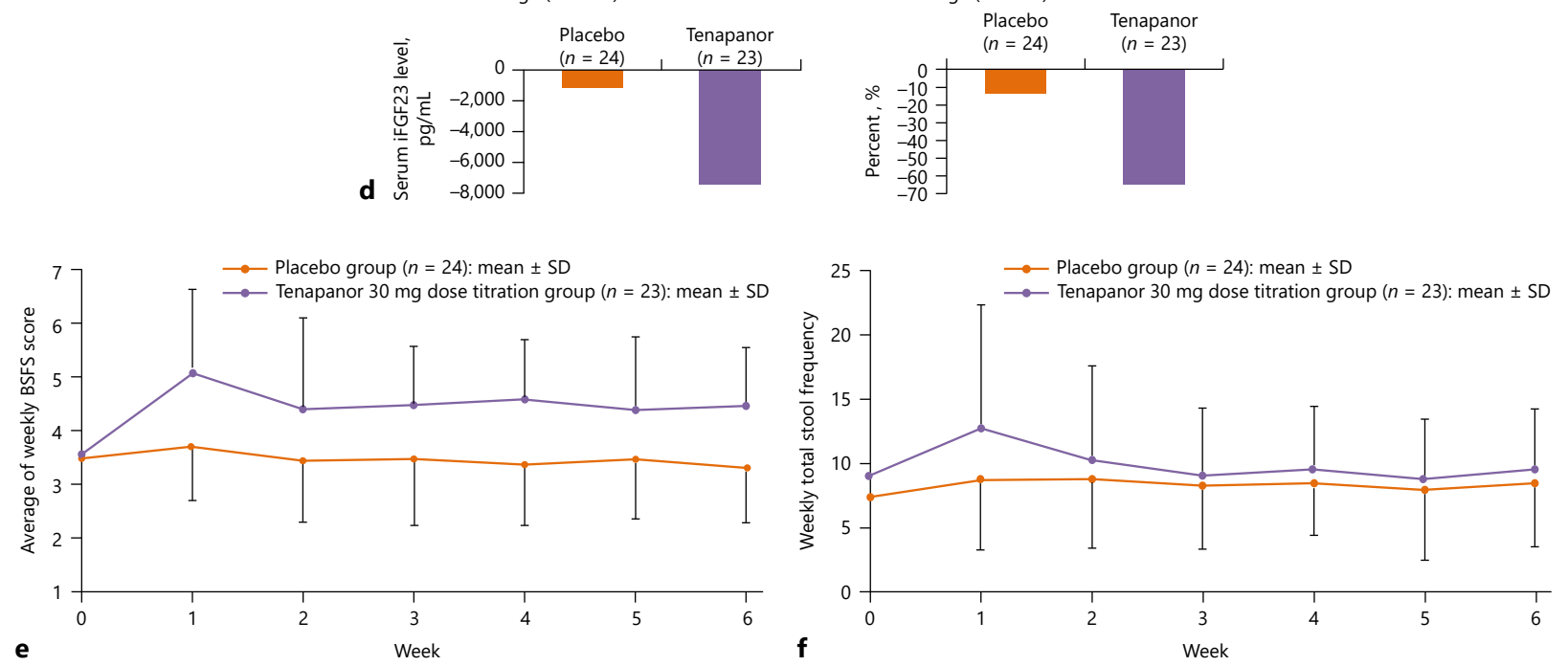

2

(For legend see next page.) 
levels when treated with tenapanor add-on to phosphate binders compared with those receiving placebo. There was a significant between-group difference of $-2.07(p<$ 0.001 ), and the mean changes from baseline to week 6 (LOCF) with placebo versus tenapanor were 0.08 versus $-1.99 \mathrm{mg} / \mathrm{dL}$, respectively. Compared with placebo, a higher proportion of tenapanor-treated patients achieved serum phosphorus target ranges $(\leq 6.0$ and $\leq 5.5 \mathrm{mg} / \mathrm{dL}$ ).

The target achievement rate of serum phosphorus level $(\leq 6.0 \mathrm{mg} / \mathrm{dL})$ at week 6 (LOCF) was $87.0 \%$ in the tenapanor group. In cases in which the target range was not achieved, serum phosphorus levels still tended to decrease. Thus, it is expected that in clinical practice, tena- panor and phosphate binders may have an additive effect resulting in improved control of serum phosphorus levels to levels within the target range in patients with poorly controlled hyperphosphatemia. Of note, half of the patients in the tenapanor group who continued treatment until week 5 had required dose reductions to $20 \mathrm{mg}$ BID, $10 \mathrm{mg} \mathrm{BID}$, and $5 \mathrm{mg}$ BID (Fig. 2c). Nevertheless, the change in serum phosphorus levels and the achievement rates of serum phosphorus target ranges were still considerable, indicating that even the lower tenapanor doses can provide serum phosphorus-lowering effects in these patients.

Diarrhea occurred in 8.3 and $65.2 \%$ of subjects treated with placebo and tenapanor, respectively, and it was the

Table 2. Overall summary of AEs and drug-related AEs by SOC and PT with an incidence $\geq 5 \%$ - safety analysis set

\begin{tabular}{lll}
\hline & Placebo & Tenapanor \\
& $(N=24), n(\%)$ & $(N=23), n(\%)$ \\
\hline Subjects with any AE & $9(37.5)$ & $18(78.3)$ \\
Serious & 0 & $1(4.3)$ \\
Death & 0 & 0 \\
Other serious AEs & 0 & $1(4.3)$ \\
Other significant AEs & 0 & $3(13.0)$ \\
Study medication discontinuation & 0 & $3(13.0)$ \\
Subjects with any drug-related AE & $2(8.3)$ & $16(69.6)$ \\
Serious & 0 & 0 \\
Death & 0 & 0 \\
Other serious AEs & 0 & 0 \\
Other significant AEs & 0 & $3(13.0)$ \\
Study medication discontinuation & 0 & $3(13.0)$ \\
Subjects with any severe AE & 0 & $1(4.3)$ \\
AEs by (SOC) and PT & $N=24$ & $N=23$ \\
(Gastrointestinal disorders) & $4(16.7)$ & $16(69.6)$ \\
Diarrhea & $4(16.7)$ & $3(65.2)$ \\
(Infections and infestations) & $2(8.3)$ & $3(13.0)$ \\
Nasopharyngitis & $1(4.2)$ & $2(8.7)$ \\
(Injury, poisoning, and procedural complications) & $2(8.3)$ & $3(13.0)$ \\
Drug-related AEs by [SOC] and PT & $N=24$ & $N=23$ \\
(Gastrointestinal disorders) & $2(8.3)$ & $16(69.6)$ \\
Diarrhea & $2(8.3)$ & $15(65.2)$ \\
\hline
\end{tabular}

Other significant AEs were defined as "all adverse events for which the study drug was discontinued." AE, treatment-emergent adverse event; PT, preferred term; SOC, system organ class.

Fig. 2. a Mean serum phosphorus levels over time (mean [SD]) mITT population. $\mathbf{b}$ Mean change in serum phosphorus levels over time (mean $[\mathrm{SD}]$ ) - mITT population. c Changes in tenapanor titration dose and number of patients who discontinued treatment by week - mITT population. $\mathbf{d}$ Mean changes and rates of changes of serum iPTH and iFGF23 levels by treatment group at the end of the study (mean [SD]) - mITT population. e Summary of average BSFS score per week - mITT population. $\mathbf{f}$ Summary of number of defecations per week - mITT population. mITT, modified intentto-treat; BSFS, Bristol Stool Form Scale; iPTH, intact parathyroid hormone; iFGF23, intact fibroblast growth factor 23; SD, standard deviation. 
most frequently occurring drug-related AE. As shown in Figure 2e, the BSFS score, which provides a clinical assessment of stools, increased in the tenapanor group after the start of treatment, but the mean value of the BSFS score was within the range of normal stool characteristics. The frequency of defecation also increased in the tenapanor group immediately after the start of treatment but was similar on average to that in the placebo group at week 6 . These findings indicate that the degree of diarrhea was not severe.

In the present study, the discontinuation rate was $30.4 \%$ in the tenapanor group, compared with $8.3 \%$ in the placebo group. The difference in discontinuation rates between groups was mainly due to the higher incidence of diarrhea in the tenapanor group. However, in all patients who discontinued treatment because of AEs, there was a trend toward lower serum phosphorus levels at the time of discontinuation compared with baseline. Therefore, prevention of the onset of diarrhea and appropriate measures for its management are important during tenapanor treatment. However, in this study, we did not identify which patients had a greater propensity to develop diarrhea, nor did we evaluate ways to prevent the onset of diarrhea or control it to ensure the continuous administration of tenapanor. Lowering the starting dose may be a strategy for controlling diarrhea; as in the single-agent study, the incidence of diarrhea was lower in the low-dose tenapanor group [14]. Thus, we need to clarify these issues in future studies.

In this study, few AEs other than diarrhea occurred, and this was consistent with studies conducted in the USA, in which tenapanor was administered as a single agent $[10,11,13,15]$. These results are also consistent with those of other ongoing tenapanor studies in Japan [14]. Further, no specific AEs were observed with the combination of tenapanor and existing phosphate binders, suggesting that the safety of tenapanor in combination with phosphate binders is acceptable.

Previous efforts have been made to develop an NPTIIb-targeted hyperphosphatemia drug that inhibits the absorption of phosphate. However, the development of this drug was discontinued [16]. Currently, tenapanor is the only drug that inhibits the NHE3 transporter of intestinal epithelial cells to reduce serum phosphorus levels rather than physically binding to phosphorus as conventional phosphate binders.

The present results showed a significant and clinically meaningful decrease in serum phosphorus levels with tenapanor compared with placebo. These findings suggest that tenapanor could be a first-in-class hyperphosphate- mia drug acting via a novel mechanism of action, which differs from conventional phosphate binders. Tenapanor is a metal-free, nonpolymer agent that is rarely absorbed by the body [13]. Additionally, we have shown that it can effectively lower serum phosphorus levels to within guideline-recommended target ranges in patients with poor control with prescribed phosphate binders. A study on the administration of phosphorus binders combined with tenapanor was recently reported [12]. Although there are limits to comparisons due to differences in study design and subject population, the efficacy and safety results of that study tended to be similar to those in this study. Therefore, tenapanor is expected to expand the range of treatment options for patients with hyperphosphatemia.

Consistent with the results of phase III studies in the USA $[11,12]$, the iFGF23 value at the end of treatment with tenapanor decreased from baseline. Although the mechanism of action is not clear, a tendency to decrease iFGF23 was observed in the tenapanor group, suggesting that the decrease in serum phosphorus level caused by tenapanor may also contribute to the reduction of iFGF23, as iFGF23 is located upstream of the regulation of serum phosphorus level in the human body.

This study had some limitations. First, we did not restrict the type of phosphate binders that patients were prescribed prior to the start of the study. Among the patients included in this study, 19 out of 23 patients were prescribed calcium carbonate as a single agent or in combination with other agents, 10 out of 23 patients were also using a single phosphate binder, and more than half of the subjects were using multiple types of phosphate binders. In contrast, the number of patients who were not prescribed calcium carbonate was relatively low. Second, the sample size may have been too small to fully elucidate the efficacy and safety of individual phosphate binders, other than calcium carbonate when combined with tenapanor. Finally, the study had a short dosing period of 6 weeks; thus, the efficacy and safety of long-term dosing should be investigated in future studies.

\section{Conclusions}

By adding tenapanor, a drug with a novel and different mechanism of action to those of conventionally used phosphate binders, CKD patients with hyperphosphatemia, whose serum phosphorus levels were poorly controlled with existing phosphate binders alone, achieved a significant decrease in serum phosphorus level compared 
with placebo. Additionally, a higher proportion of tenapanor-treated patients achieved serum phosphorus targets as recommended in the current treatment guideline compared with placebo. No new safety signals were raised, and tenapanor was generally well tolerated. The present findings indicate that tenapanor may satisfy the unmet need for serum phosphorus control in hemodialysis patients with hyperphosphatemia who have poor phosphorus management with prescribed phosphate binders.

\section{Acknowledgement}

The authors wish to thank Keyra Martinez Dunn, MD, of Edanz Pharma for providing medical writing support, which was funded by Kyowa Kirin Co., Ltd.

\section{Statement of Ethics}

The ethical review boards of the participating sites approved the protocol and related documents. The study adhered to Good Clinical Practice guidelines and the Declaration of Helsinki, as well as local laws and regulations. All patients provided informed consent before the conduct of any study procedures. The study was registered in Clinicaltrials.gov under the identifier NCT03864445.

\section{Conflict of Interest Statement}

T.S. has received personal fees from Kyowa Kirin Co., Ltd. during the conduct of the study and outside the submitted work. M.F. has received personal fees from Kyowa Kirin Co., Ltd., Ono Pharmaceutical Co. Ltd., Torii Pharmaceutical Co. Ltd., and Kissei Pharmaceutical Co. Ltd. and grants from Bayer Yakuhin Ltd. dur- ing the conduct of the study. T.A. has received personal fees from Kyowa Kirin Co., Ltd. during the conduct of the study and personal fees from Astellas, Bayer Yakuhin Ltd., Kissei Pharmaceutical Co. Ltd., Ono Pharmaceutical Co. Ltd., Fuso Pharmaceutical Industries Ltd., Torii Pharmaceutical Co. Ltd., GlaxoSmithKline, J.T. Pharmaceuticals, Nipro Corporation, Otsuka, Sanwa Chemical, and Chugai Pharmaceutical Co. Ltd. outside the submitted work. Y.U., K.I., and H.K. are employees of Kyowa Kirin Co., Ltd.

\section{Funding Sources}

This research was funded by Kyowa Kirin Co., Ltd.

\section{Author Contributions}

T.S. contributed to the study design and data analysis, drafted the main manuscript, and provided final approval for submission. Y.U. contributed to the study design and data analysis, reviewed the manuscript critically for important intellectual content, and provided final approval for submission. K.I. contributed to the study design and data analysis, reviewed the manuscript critically for important intellectual content, and provided final approval for submission. H.K. reviewed the manuscript critically for important intellectual content and provided final approval for submission. M.F. contributed to the study design and data analysis, reviewed the manuscript critically for important intellectual content, and provided final approval for submission. T.A. contributed to the study design and data analysis, reviewed the manuscript critically for important intellectual content, and provided final approval for submission.

\section{Availability of Data and Material}

Due to the nature of this research, participants of this study did not agree for their data to be shared publicly, so supporting data are not available.

\section{References}

1 GBD Chronic Kidney Disease Collaboration. Global, regional, and national burden of chronic kidney disease, 1990-2017: a systematic analysis for the global burden of disease study 2017. Lancet. 2020 Feb 29;395(10225):709-33.

2 Hannedouche T, Fouque D, Joly D. (Metabolic complications in chronic kidney disease: hyperphosphatemia, hyperkalemia and anemia). Nephrol Ther. 2018 Nov;14(6 Supplement):6S17-25.

3 Marcuccilli M, Chonchol M, Jovanovich A. Phosphate binders and targets over decades: do we have it right now? Semin Dial. 2017 Mar;30(2):134-41.

4 Vervloet MG, van Ballegooijen AJ. Prevention and treatment of hyperphosphatemia in chronic kidney disease. Kidney Int. 2018 May; 93(5):1060-72.

Add-On Effects of Tenapanor in Hemodialysis Patients with Hyperphosphatemia
5 Cozzolino M, Ciceri P, Galassi A, Mangano M, Carugo S, Capelli I, et al. The key role of phosphate on vascular calcification. Toxins. 2019 Apr 9;11(4):213.

6 Floege J. Phosphate binders in chronic kidney disease: a systematic review of recent data. J Nephrol. 2016 Jun;29(3):329-40.

7 Fukagawa M, Yokoyama K, Koiwa F, Taniguchi M, Shoji T, Kazama JJ, et al. Clinical practice guideline for the management of chronic kidney disease-mineral and bone disorder. Ther Apher Dial. 2013 Jun;17(3): 247-88.

8 Cernaro V, Santoro D, Lacquaniti A, Costantino G, Visconti L, Buemi A, et al. Phosphate binders for the treatment of chronic kidney disease: role of iron oxyhydroxide. Int $\mathrm{J}$ Nephrol Renovasc Dis. 2016;9:11-9.
9 King AJ, Siegel M, He Y, Nie B, Wang J, KooMcCoy S, et al. Inhibition of sodium/hydrogen exchanger 3 in the gastrointestinal tract by tenapanor reduces paracellular phosphate permeability. Sci Transl Med. 2018 Aug 29; 10(456):eaam6474.

10 Johansson S, Rosenbaum DP, Knutsson M, Leonsson-Zachrisson M. A phase 1 study of the safety, tolerability, pharmacodynamics, and pharmacokinetics of tenapanor in healthy Japanese volunteers. Clin Exp Nephrol. 2017 Jun;21(3):407-16.

11 Block GA, Rosenbaum DP, Yan A, Chertow GM. Efficacy and safety of tenapanor in patients with hyperphosphatemia receiving maintenance hemodialysis: a randomized phase 3 trial. J Am Soc Nephrol. 2019 Apr; 30(4):641-52. 
12 Rosenbaum DYY. Efficacy of tenapanor for the control of serum phosphorus in patients with ckd on dialysis: novel mechanism of action allows for both monotherapy and dual mechanism approach. National kidney foundation spring clinical meetings. New Orleans, Louisiana: National Kidney Foundation; 2020.
13 Block GA, Rosenbaum DP, Leonsson-Zachrisson M, Åstrand M, Johansson S, Knutsson $\mathrm{M}$, et al. Effect of tenapanor on serum phosphate in patients receiving hemodialysis. J Am Soc Nephrol. 2017 Jun;28(6):1933-42.

14 Inaguma DSY, Takanuma M, Kanda $\mathrm{H}, \mathrm{Fu}-$ kugawa M, Akizawa T. Dose-response efficacy and tolerability of tenapanor on hyperphosphatemia in japanese hemodialysis patients: results of a randomized phase 2 study. J Am Soc Nephrol. 2020.
15 Rosenbaum DP, Yan A, Jacobs JW. Pharmacodynamics, safety, and tolerability of the NHE3 inhibitor tenapanor: two trials in healthy volunteers. Clin Drug Investig. 2018 Apr;38(4):341-51.

16 Larsson TE, Kameoka C, Nakajo I, Taniuchi Y, Yoshida S, Akizawa T, et al. NPT-IIb inhibition does not improve hyperphosphatemia in CKD. Kidney Int Rep. 2018 Jan;3(1):73-80. 